\title{
Magnetic susceptibility of n- and p-Si single crystals containing thermodonors
}

\author{
V.M. Babich ${ }^{1}$, M.M. Luchkevych ${ }^{2,3}$, V.M. Tsmots ${ }^{3}$ \\ ${ }^{1}$ Institute of Semiconductor Physics, NAS of Ukraine, 41, prospect Nauky, \\ 03028 Kyiv, Ukraine; e-mail: babich@isp.kiev.ua \\ ${ }^{2}$ Institute of Nuclear Researches, NAS of Ukraine, 47, prospect Nauky, 03028 Kyiv, Ukraine \\ ${ }^{3}$ Drohobych Ivan Franko State Pedagogical University, 24, Franko str., \\ 82100 Drohobych, Ukraine; e-mail:lab_mtme@drohobych.net
}

\begin{abstract}
Using a series of experimental methods (Hall effect, electron paramagnetic resonance and magnetic susceptibility $(\mathrm{MS}, \chi)$ ) comparison has been made for the kinetics of formation inherent to different types of thermodonors (TDs) (doubly-charged and shallow ones) under thermal treatments (TT) at $450{ }^{\circ} \mathrm{C}$ with the kinetics of paramagnetic center accumulation in the samples obtained by the MS method. The absence of correlation between these dependences has been shown. The dependences $\chi(H)$ obtained both at 300 and $77 \mathrm{~K}$ that have orientation and paramagnetic component determine neither of these two kinds of TD-centers as it was in the case of $450{ }^{\circ} \mathrm{C}$ TT for $p$-Si(B) samples where the $\chi^{\mathrm{par}}$ component is determined by the concentration of deep TDs $\left(E_{i} \geq 0.2 \mathrm{eV}\right)$. The $\chi(H)$ value in the $n-\mathrm{Si}(\mathrm{P})$ samples after TT at $450{ }^{\circ} \mathrm{C}$ is determined, evidently, by intermediate non-stable complexes, as well as by those formed during rapid cooling the samples
\end{abstract}

Keywords: diamagnetism, paramagnetism, magnetic susceptibility, thermodonors, thermal treatment.

Manuscript received 05.03.10; accepted for publication 02.12.10; published online 30.12.10.

\section{Introduction}

It is known that due to decomposition of saturated solid oxygen solutions under thermal treatment (TT) of the Czochralski-grown $\mathrm{Si}$ single crystals, in the wide temperature interval $\left(400-1200^{\circ} \mathrm{C}\right)$ a series of microdefects is formed, which leads to a significant change in electrical, physical and structural properties of the material. Specifically, the change in electrical and physical properties of $\mathrm{Si}$ is enhanced by the process of formation of thermodonors (TD) and thermoacceptors (TA) of oxygen nature during $400-800{ }^{\circ} \mathrm{C}$ TT [1].

Three types of TD-I formed using TT of oxygencontaining $\mathrm{Si}$ crystals in the $400-500{ }^{\circ} \mathrm{C}$ temperature range [1] have been determined by numerous experiments: 1) STDs - shallow thermodonors $\left.\left(E_{i} \approx 31.3-37.4 \mathrm{meV}\right) ; 2\right)$ doubly-charged TDs $\left(E_{i} \approx 53.0\right.$ $69.3 \mathrm{meV}$ for the $\mathrm{TD}^{0}$ state and $E_{i} \approx 127.9-156.3 \mathrm{meV}$ for the $\mathrm{TD}^{+}$state); 3) DTD - deep TD with $E_{i} \geq 200.0 \mathrm{meV}$.
TT of the samples at $600-800{ }^{\circ} \mathrm{C}$ leads to formation of a set of singly-charged TD-II ("new" TD) with ionization energies of $10-300 \mathrm{meV}[2,3]$.

TT of the samples in this temperature range also leads to formation of thermoacceptors beside TD-II. One type of TA is Si-nk centers that are deep electron traps with $E_{C}=0.06 \mathrm{eV}$ [4]. According to [4], this type of TA is related with the dislocation dipoles that appear near ribbon-like precipitates. As determined by DLTS methods, a whole series of TA-centers with $E_{T A}$ $E_{V} \approx 0.4-0.6 \mathrm{meV}$ appears at $500-900{ }^{\circ} \mathrm{C}$ TT of oxygencontaining Si samples [5].

Studies of the electron spin resonance (ESR) spectra for oxygen-containing Si samples undergone TT in both temperature ranges allowed to determine a series of paramagnetic centers that correspond to TD-I (these are spectra NL 8, NL 9, NL 10 in accord with the classification [6]), as well as a set of spectra in a wide range of g-factors that correspond to TD-II. These experiments where made at $T \leq 50 \mathrm{~K}$. Evidently, the TD- 
centers and accompanying TA-centers that are characterized by paramagnetic properties can be studied at higher temperatures using, for example, informative methods of measurements of static magnetic susceptibility (MS) by using the Faraday method.

The authors in [8], interpreting the dependence of MS on magnetic field $H$ for the $n$-Si(P) samples undergone TT at $450{ }^{\circ} \mathrm{C}$, concluded that there exists the magnetic ordering phenomenon of cooperative type for doubly-charged TDs caused by heterogeneous distribution of interstitial oxygen inside the crystal. The authors of the work [9] made a similar conclusion with reference to A-centers formed by electron irradiation of oxygen-containing Si samples. In [10] studying the MS of oxygen-containing $p$ - $\mathrm{Si}(\mathrm{B})$ samples grown in "vacancy" mode undergone $450{ }^{\circ} \mathrm{C} \mathrm{TT}$, independence of $\chi$ from $H$ both at 300 and $77 \mathrm{~K}$ has been revealed. This fact shows the non-cooperative character of the revealed magnetism. The authors explained the appearance of such paramagnetic properties by the presence of DTDs in the samples. We can also note that in the latter experimental samples doubly-charged TDs existed (roughly of the same concentration as in $n-\mathrm{Si}(\mathrm{P})$ material which had been studied in [8]) together with DTDs. However, no nonlinear dependences $\chi(H)$ have been observed in them. These experimental facts show that MS in Si crystals in the presence of doubly-charged TDs cannot be definitely explained. This induced us to make more detailed experiments to study MS in oxygencontaining $\mathrm{Si}$ crystals after low temperature TT $\left(\approx 450^{\circ} \mathrm{C}\right)$ of varying durations.

\section{Experimental}

For the experiment, we chose Czochralski-grown $n$-Si(P) material $\left(\rho_{300 \mathrm{~K}} \approx 30 \mathrm{Ohm} \cdot \mathrm{cm}, \quad n_{e} \approx 1 \cdot 10^{14} \mathrm{~cm}^{-3}\right)$. The amount of background impurities in the crystal was as follows: interstitial oxygen $\left[\mathrm{O}_{i}\right] \approx 9.5 \cdot 10^{17} \mathrm{~cm}^{-3}$ and substitution carbon $\left[\mathrm{C}_{s}\right] \approx 3 \cdot 10^{16} \mathrm{~cm}^{-3}$. The samples were heated at $450{ }^{\circ} \mathrm{C}$ (in the air) for 5-500 hours. Each sample was undergone TT during 5, 10, 20, 110, 200, 300 and 500 hours and MS at 300 and $77 \mathrm{~K}$, as well as temperature dependences of the charge concentration by the Hall effect in the wide temperature range $(20-300 \mathrm{~K})$ were measured. The study of static magnetic susceptibility $\chi(H)$ of the samples was made using the equipment described in detail in [7]. Its sensitivity was $1 \cdot 10^{15} \mu_{\mathrm{B}} / \mathrm{cm}^{3}$. The maximum relative error was close to $3 \%$. For the measurements, $2 \times 3 \times 10 \mathrm{~cm}$ samples were cut out, then polished by M-7 powder and by diamond paste with the grain size of $2-3 \mu \mathrm{m}$ and etched in polishing etchant.

\section{Results and discussion}

Fig. 1 shows the temperature dependences of the concentration of conduction electrons for the samples thermally treated at $450{ }^{\circ} \mathrm{C}$ for various periods.

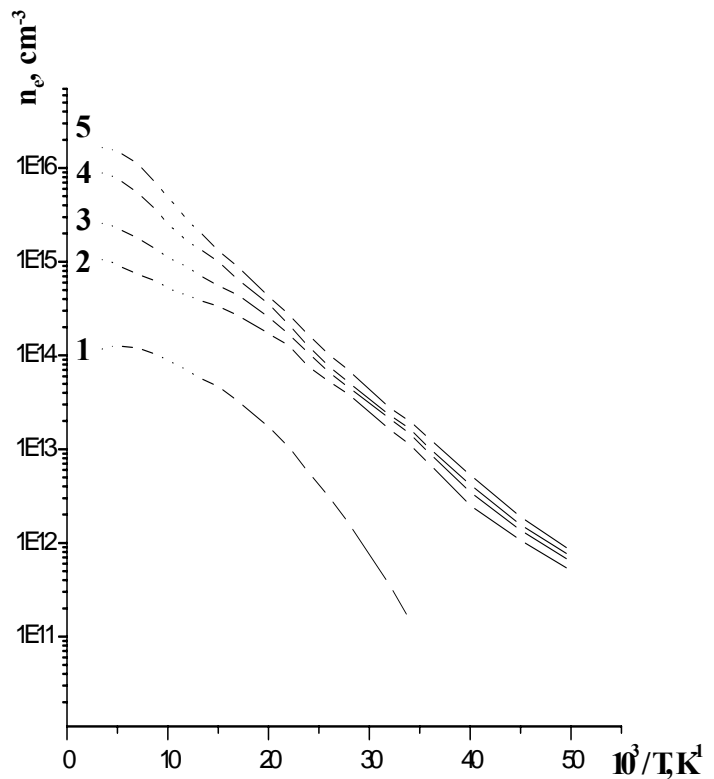

Fig. 1. Temperature dependences of the concentration of conduction electrons in the initial (without TT) sample ( 1 ) and samples treated at $450{ }^{\circ} \mathrm{C}$ for: $2-5 ; 3-10 ; 4-50 ; 5-500$ hours.

Our interpretation of these Hall-effect measurements demonstrates that in this $n$-Si(P) material under this TT TDs-centers of two types are formed: doubly-charged (which appear in the range $50-300 \mathrm{~K}$ in Fig. 1) and singly-charged shallow TD-centers (STD), which determine the slope in the dependences $n_{e}=f\left(10^{3} / T\right)$ in the temperature range $20-50 \mathrm{~K}$ and possess the thermal ionization energy $E_{i} \approx 30 \mathrm{meV}$.

Determination of $n_{e}$ concentration at $300 \mathrm{~K}$ for all the samples undergone $\mathrm{TT}$ at $450{ }^{\circ} \mathrm{C}$ show the (total) kinetics of TD-center formation in the course of the above TT (curve 1, Fig. 2). The study of ESR spectra for these samples at low temperatures $(\approx 20-30 \mathrm{~K})$ allowed to determine the kinetics of STD-centers accumulation (curve 2, Fig. 2). The concentration of doubly-charged TDs was determined for corresponding TT duration $t$ according to the formula $N_{T D}=\left(n_{e}-N_{E S R}\right) / 2\left(\mathrm{~cm}^{-3}\right)$, it is shown in Fig. 2 as the curve 2. The maximum total concentration of TD-centers is reached after 300-500 hours of TT and equals $1.5 \cdot 10^{16} \mathrm{~cm}^{-3}$. STDs made up only $\approx 10 \%$ of the total amount of TDs. Doubly-charged TDs in the $n$ - $\mathrm{Si}(\mathrm{P})$ crystals in the ESR spectra is not usually observed as shown earlier [1]. The observation of the doubly-charged TD spectra is possible in the singly-ionized state of $\mathrm{TDs}^{+}$as NL 8 spectra in the case of presence of doping acceptor impurities or acceptor Acenters of oxygen nature after $\gamma$ - or electron irradiation in the samples with the doubly-charged TDs.

The dependences of the static magnetic susceptibility on the magnetic field intensity $\chi(H)$ for the samples undergone $\mathrm{TT}$ at $450{ }^{\circ} \mathrm{C}$ are shown in Fig. 3. The measurements were made in the magnetic fields of 0 to $4.5 \mathrm{kOe}$ at $77 \mathrm{~K}$. 


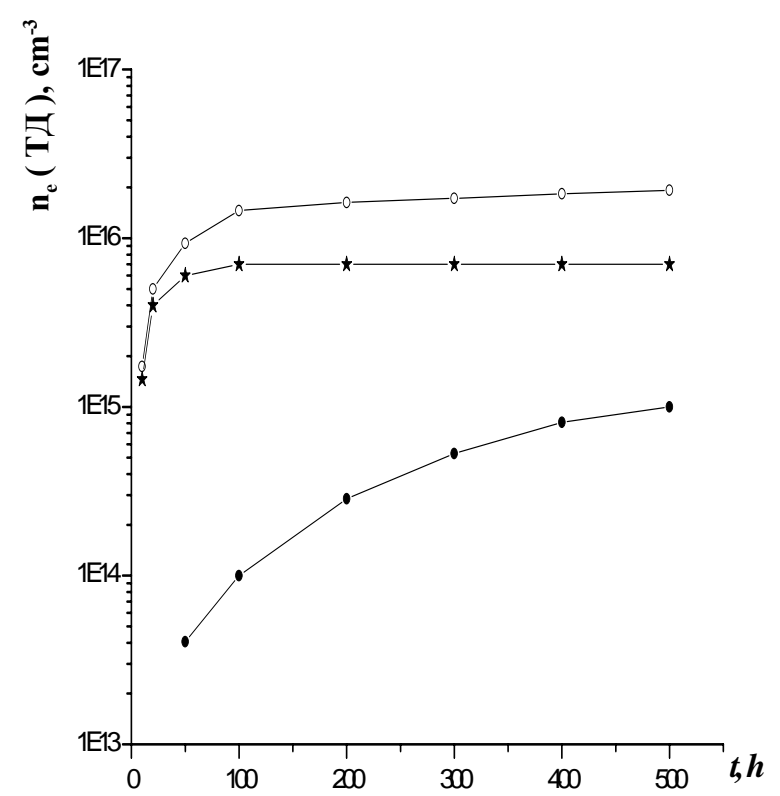

Fig. 2. The dependence of the concentration of conduction electrons at $300 \mathrm{~K}$ in $n$ - $\mathrm{Si}(\mathrm{P})$ samples on TT duration at $450{ }^{\circ} \mathrm{C}(1)$ and on the kinetics of accumulation of doublycharged TDs (2) and STDs (3, obtained from ESR spectra).

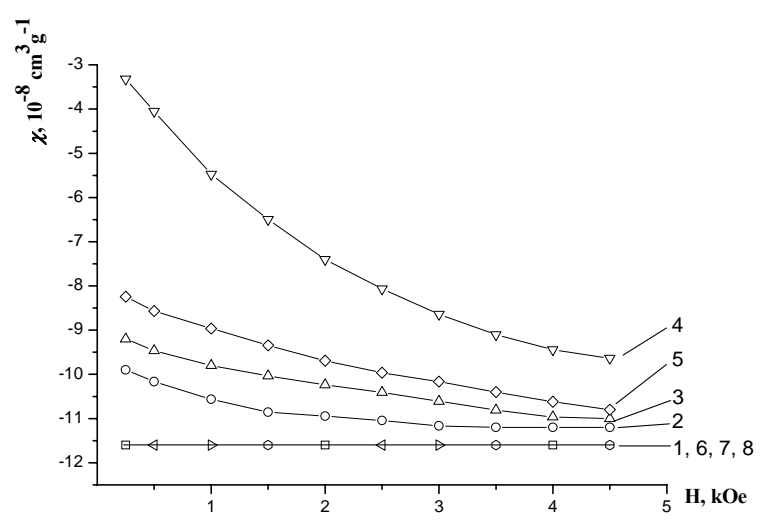

Fig. 3. The dependences $\chi(H)$ for $n$-Si(P) samples thermally treated at $450{ }^{\circ} \mathrm{C}: 1$ stands for the initial sample (without TT); $2-5 ; 3-10 ; 4-20 ; 5-100 ; 6-200 ; 7-300 ; 8-500$ hours.

Unlike the results of [8] where nonlinear dependences of $\chi(H)$ were not observed (for two thermally treated samples, they concurred with the dependence for the initial sample), in our experiment these dependences were revealed (curves 1-5, Fig. 3). Similar results were observed at the temperature $77 \mathrm{~K}$. The nonlinear dependences of $\chi(H)$ were not observed only in the samples thermally treated for a long time (from 200 to 500 hours, curves $6,7,8$ Fig. 3). The values $\chi$ in these samples were similar to those for the initial sample (without TT) (curve 1). The nonlinear dependence $\chi(H)$ is observed for the samples thermally treated for up to 100 hours (curves 2-5) and can be presented in the following way:

$\chi_{\exp }=\chi_{L}+\chi^{\mathrm{par}}+\chi^{\text {ord }}(H)$, where $\chi_{L}$ is the susceptibility of the grate of the initial sample, $\chi^{\mathrm{par}}$ - paramagnetic constituent of MS that is responsible for the decrease of diamagnetism, $\chi^{\text {ord }}(H)$ constituent of MS caused by the magnetic ordering and responsible for the appearance of nonlinear dependences $\chi(H)$ in the samples annealed at $450^{\circ} \mathrm{C}$.

The constituent $\chi^{\mathrm{par}}$ is determined at $H=4.5 \mathrm{kOe}$ (the accumulation area of $\chi(H)$ dependences). For the sample with a number $n$, the paramagnetic constituent is determined as the difference: $\chi_{n}{ }^{\mathrm{par}}=\chi_{n}(4.5)-\chi_{L}$. If in this way we define $\chi^{\text {par }}$ for all the samples thermally treated within the range of durations $0-500$ hours, we obtain the dependence shown in Fig. 4.

The comparison of the dependences $\chi^{\mathrm{par}}=f\left(t_{\mathrm{ann}}\right)$ on MS shown in Fig. 4 with the dependences 2 and 3 in Fig. 2 that characterizes the kinetics of accumulation of doubly-charged and singly-charged TD-centers at TT of the samples $n$ - $\mathrm{Si}(\mathrm{P})$ with the oxygen demonstrates the lack of correlation between the dependence $\chi^{\mathrm{par}}=f(t)$ (Fig. 4) and any dependences Fig. 2. It proves the fact that the MS experiments both at 300 and $77 \mathrm{~K}$ in $n-\mathrm{Si}(\mathrm{P})$ samples thermally treated at $450{ }^{\circ} \mathrm{C}$ contain STDs and doubly-charged TD-centers and do not allow the study of the kinetics of accumulation for two types of these TD-centers unlike the case with STDs in $p$-Si(B) crystal [10]. Therefore, the issue of the type of paramagnetic centers, we deal with in the MS experiments in $n-\operatorname{Si}(\mathrm{P})$ samples thermally treaded at $450{ }^{\circ} \mathrm{C}$ and the determination of their accumulation kinetics, remains open (Fig. 4). Besides, it is difficult to explain the nonlinear character of the dependences of $\chi(H)$ that demonstrates the presence of magnetic ordering of the centers without taking into consideration the authors' thoughts in [8] about the role of doubly-charged TDs in the singly-ionized state of $\mathrm{TDs}^{+}$, however almost all the doubly-charged TDs are ionized and remain in the state $\mathrm{TDs}^{++}$at $300 \mathrm{~K}$.

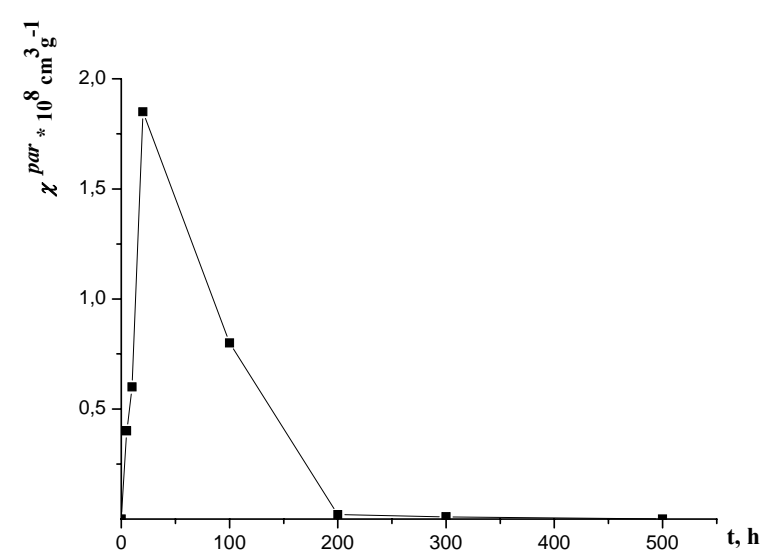

Fig. 4. The kinetics of accumulation of paramagnetic centers with the time of TT that were obtained from the experimental curves in Fig. 3 at $H=4.5$ kOe. 


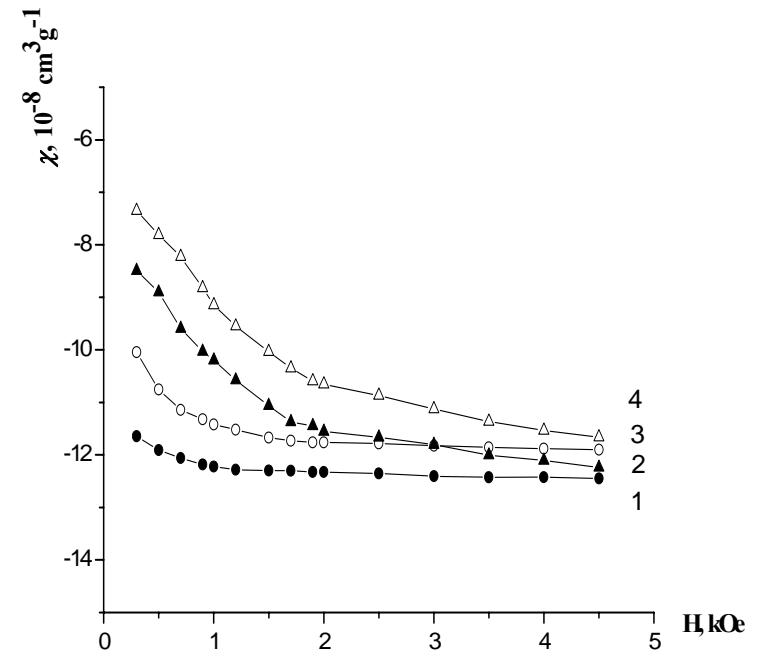

Fig. 5. The MS dependences for $n-\operatorname{Si}(\mathrm{P})$ sample thermally treated at $450{ }^{\circ} \mathrm{C}$ for 5 hours: $1,2-T_{\text {meas }}=77 \mathrm{~K} ; 3,4-T_{\text {meas }}=$ $300 \mathrm{~K} ; 1,3$ - slow cooling; 2, 4- rapid cooling (hardening).

It should be noted that the nonlinear character of the dependences of $\chi(H)$ that show the magnetic ordering of certain paramagnetic centers was observed not only in $n$-Si(P) samples at the presence of doubly-charged TDs but also in the case of these crystals undergone TT in the temperature range of $650-1100{ }^{\circ} \mathrm{C}$ [11] and also in the samples plastically deformed at the temperatures 650 $700{ }^{\circ} \mathrm{C}[12]$. In all these cases, the main stage of sample preparation for measurements is the thermal treatment at a certain temperature. At the same time, it is known that under certain conditions of the thermally treated samples cooling from the TT temperature to the ambient temperature, the so-called hardening centers form in the samples (including those with paramagnetic properties). Is not the appearance of the MS constituent $\chi^{\text {ord }}(H)$ related with these centers? Do such centers appear in conditions of our experiments at TT near $450{ }^{\circ} \mathrm{C}$ ? The answers to these questions have been given by the following experiments.

The MS dependences for the $n$-Si(P) sample thermally treated for 5 hours at $450{ }^{\circ} \mathrm{C}$ but cooled in various ways to the ambient temperature are shown in Fig. 5: in the first case, the sample was cooled together with the oven, while in the second case, the same sample was again heated for $10 \mathrm{~min}$ at $450{ }^{\circ} \mathrm{C}$ and then hardened by putting the sample into oil. Prominent is the fact of a sharp increase of the MS constituent $\chi^{\text {ord }}(H)$ for the rapidly cooled sample in the weak magnetic field $H \approx 0.3 \mathrm{kOe}$ (compare the curves 1, 2, 3 and 4 obtained at two different temperatures ( 77 and $300 \mathrm{~K})$ of the experiment that attracts the attention). At the same time, the decrement of the MS constituent $\chi^{\text {par }}$ (at $4.5 \mathrm{kOe}$ ) for the rapidly cooled sample compared with the slowly cooled one is insignificant (compare the same curve 1, 2, 3 and 4 at $4.5 \mathrm{kOe}$ ) and is roughly equal for both temperatures of the measurements.
These experiments also show that neither the doubly-charged TDs nor singly-charged STDs are related to the nonlinear dependences $\chi(H)$, as the same quantity of TD-centers (approximately $10^{15} \mathrm{~cm}^{-3}$ ) that formed due to annealing for 5 hours remained in the samples (both slowly and rapidly cooled). Therefore, we deal with formation of the hardening paramagnetic centers that is shown by the orientation dependences $\chi(H)$. Of course, the appearance of these centers in the thermally treated $\mathrm{Si}$ samples within the higher temperature range $\left(650-1100^{\circ} \mathrm{C}\right)$ as in [11] and at the single-axis plastic deformation of $n$-Si(P) samples [12] should be expected. That is why, interpreting the experimental MS data in all these cases, the probability of appearance of such paramagnetic centers should be taken into account and their share in the measured MS value should be determined.

\section{Conclusions}

Thus, our comparison of the kinetics of TD-center formation (doubly-charged TDs and singly-charged STDs) formed by $\mathrm{TT}$ at $450{ }^{\circ} \mathrm{C}$ in the oxygen-containing $n$-Si(P) crystals obtained due to Hall effect and ESR with the kinetics of the paramagnetic center accumulation in them obtained (at 300 and $77 \mathrm{~K}$ ) by the MS method shows that these dependences are not correlated. The dependences $\chi(H)$ obtained both at 300 and $77 \mathrm{~K}$ by the MS method with $\left(\chi^{\text {ord }}\right)$ orientation and paramagnetic constituent $\left(\chi^{\mathrm{par}}\right)$ are not determined by either of these two types of TD-centers. In particular, the $\chi^{\text {par }}$ constituent increases first with the increased annealing duration of the samples within the range of 0 500 hours, then passes through the maximum and decreases to zero at high $t \geq 200$ hours, while the kinetics of TD formation of these two types increases and tends to saturation. On the contrary, in $p$-Si(B) material where deep TD-centers $\left(E_{i} \geq 200 \mathrm{meV}\right)$ are formed by annealing at $450{ }^{\circ} \mathrm{C}$, the $\chi^{\mathrm{par}}$ constituent is correlated to the concentration of these centers [10].

The appearance of the dependence of $\chi(H)$ on the magnetic field in $n$-Si(P) samples thermally treated at $450{ }^{\circ} \mathrm{C}$ can be explained by formation of different kinds of intermediate unstable (bistable) complexes of oxygen (or other) nature during $\mathrm{TT}$ at $450{ }^{\circ} \mathrm{C}$, which with the increase of annealing duration are reformed into the stable thermodonor or neutral impurity complex changing their paramagnetic properties. One of the variety of such complexes are the complexes formed under the condition of rapid cooling the crystals from the temperature of TT $450{ }^{\circ} \mathrm{C}$ to the ambient temperature leading to the appearance of the observed paramagnetism.

\section{References}

1. V.M. Babich, N.I. Bletskan, Ye.F. Venger, Oxygen in Silicon Single Crystals. Kyiv, Interpress Ltd., 1997 (in Russian). 
2. A. Kanamori, M. Kanamori // J. Appl. Phys. 50(12), p. 8095-8101 (1979).

3. V.M. Babich, N.P. Baran, Yu.P. Dotsenko et al. // Ukrainskii fizich. zhurnal 33(4), p. 593-598 (1988), in Russian.

4. V.M. Babich, N.P. Baran, A.A. Bugai et al. // Zhurnal eksperiment. teoretich. fiziki 94(8), p. 319330 (1988), in Russian.

5. K. Schmalz, F.-G. Kirscht, K. Klose et al. // Phys. status solidi 100(2), p. 567-582 (1987).

6. S.H. Muller, M. Sprenger, E.G. Sieverts, C.A.J. Ammerlaan // Solid State Communs. 25(12), p. $987-990$ (1978).

7. V.M. Tsmots, I.S. Pan'kiv, L.I. Pan'kiv et al. // Patent of Ukraine № 77284 from 15.11.2006.
8. V.B. Neymash, T.R. Sagan, V.M. Tsmots et al. // Ukrainskii fizich. zhurnal 37(3), p. 437-441 (1992), in Russian.

9. A.N. Kabaldin, V.B. Neymash, V.M. Tsmots et al. // Ukrainskii fizich. zhurnal 10(3), p. 218-221 (1995), in Russian.

10. V.M. Babich, M.M. Luchkevich, Yu.V. Pavlovskyy, V.M. Tsmots // Semiconductor Physics, Quantum Electronics and Optoelectronics 11(3), p. 226-229 (2008).

11. N.N. Novikov, B.D. Patsai, V.M. Tsmots et al. // Zhurnal fizych.doslidzhen' 9(4), p. 319-324 (2005), in Ukrainian.

12. V.M. Tsmots, P.G. Litovchenko, Yu.V. Pavlovskyy et al. // Ukrainsky fizychny zhurnal 54(10), p. 10091015 (2009) in Ukrainian. 